Pacific Journal of Mathematics

A STRONG MAXIMUM PRINCIPLE FOR WEAKLY 


\title{
A STRONG MAXIMUM PRINCIPLE FOR WEAKLY SUBPARABOLIC FUNCTIONS
}

\author{
AvNer Friedman
}

Introduction. It has been proved by E. Hopf [3], over thirty years ago, that solutions of second order elliptic equations satisfy the maximum principle. A similar principle, well known for solutions of the heat equation, has been, relatively recently, extended to second order parabolic equations by Nirenberg [5]. In various problems, such as in solving the Dirichlet problem by the methods of Poincaré and Perron, subsolutions have been introduced and the maximum principle has been extended to such functions. In the elliptic case (see [6]) the subsolutions used are continuous, whereas in the parabolic case, they may have certain discontinuities (see [2]). In the elliptic case, they are called $L$-subharmonic or subelliptic functions. Likewise, in the parabolic case, we call them $L$-subcaloric or subparabolic functions; $L$ is the elliptic or the parabolic operator.

Recently, Walter Littman [4] has generalized the concept of $L$-subharmonic functions to include measurable integrable functions. This generalization is obtained by expressing the condition $L u \geqq 0$ in an integrated form, namely, $\int u L^{*} v d x \geqq 0$ for any twice differentiable $v \geqq 0$ with compact support, $L^{*}$ being the adjoint of $L$. He then established the maximum principle in the following sense: If an $L$-subharmonic function assumes its essential supremum at a point of continuity, then it is equal to a constant almost everywhere.

The purpose of this paper is to prove a similar result for measurable $L$-subcaloric functions. The general outline of the proof is similar to that of Littman's method. However, the crucial step in the proof is the construction of two kernal functions with certain required properties. Our construction is entirely different from that of Littman.

In $\S 1$ we state some definitions and the results of the paper. In $\S 2$ we prove Lemma 2 . In $\S 3$ we recall some properties of fundamental solutions. These are used in $\S 4$ to prove Lemma 1 . Lemmas 1,2 immediately yield the maximum principle.

1. Statement of the results. Consider the differential operators

$$
L u \equiv \sum_{i, j=1}^{n} a_{i j}(x, t) \frac{\partial^{2} u}{\partial x_{i} \partial x_{j}}+\sum_{i=1}^{n} a_{i}(x, t) \frac{\partial u}{\partial x_{i}}+a(x, t) u-\frac{\partial u}{\partial t}
$$

Received March 21, 1960. Prepared under Contract Nonr 710(16) (NR 044 004) between the Office of Naval Research and the University of Minnesota. 


$$
L^{*} u \equiv \sum_{i, j=1}^{n} b_{i j}(x, t) \frac{\partial^{2} u}{\partial x_{i} \partial x_{j}}+\sum_{i=1}^{n} b_{i}(x, t) \frac{\partial u}{\partial x_{i}}+b(x, t) u+\frac{\partial u}{\partial t}
$$

where $L^{*}$ is the adjoint of $L$ (thus, $b_{i j}=a_{i j}$, etc.). Throughout this paper it will always be assumed that:

$$
a_{i j}, \frac{\partial}{\partial x_{k}} a_{i j}, \frac{\partial^{2}}{\partial x_{k} \partial x_{m}} a_{i j}, a_{j}, \frac{\partial}{\partial x_{k}} a_{i}, a
$$

are Hölder continuous (exponent $\alpha$ ) in $(x, t)$ which varies in a bounded domain $D$, and that

$$
a \leqq 0 \text { in } D, \Sigma a_{i j} \xi_{i} \xi_{j} \geqq A_{0} \Sigma \xi_{i}^{2} \text { in } D\left(A_{0}>0\right)
$$

for any real vector $\xi$.

Definition. A bounded measurable function $u(x, t)$ in $D$ is called weakly L-subcaloric (or simply, weakly subparabolic when there is no confusion about the $L$ ) if for any compact subdomain $E$ of $D$ with piecewise smooth boundary (so that Green's formula holds)

$$
\iint_{E} u(x, t) L^{*} v(x, t) d x d t \geqq 0
$$

for any function $v(x, t)$ satisfying the following properties:

(i) $v \geqq 0$ in $E$,

(ii) $v, \partial v / \partial v_{i}, \partial^{2} v / \partial x_{i} \partial x_{j}, \partial v / \partial t$ are continuous in $E$ and vanish on the boundary $\partial E$ of $E$.

We note that, for the establishment of the maximum principle below, it is enough that (1) holds only for some special types of domains, namely, for cylindrical domains and for certain sections of paraboloids.

Definitions. For any point $P\left(x^{0}, t^{0}\right)$ in $D$, we denote by $C(P)$ the set of all points $\left(x^{1}, t^{1}\right)$ in $D$ such that there exists a differentiable curve connecting $\left(x^{0}, t^{0}\right)$ to $\left(x^{1}, t^{1}\right)$ and along which the $t$-coordinate is non-increasing. A function $u(x, t)$ is said to be continuous from below at a point $P=\left(x^{0}, t^{0}\right)$ if $u$, as a function in $C(P)$, is continuous at $P$ in the usual sense. By a neighborhood-from-below of a point $P$ we mean the intersection of a neighborhood of $P$ with $C(P)$.

Our purpose is to prove the following theorem.

TheOREM. Let $u$ be a weakly L-subcaloric in $D$. If $u$ assumes its essential supremum $M($ in $D)$ at a point $P=\left(x^{0}, t^{0}\right)$ at which $u$ is continuous from below, and if $M \geqq 0$, then $u=M$ almost everywhere in $C(P)$.

As in [4], the proof follows immediately once we have established the following lemmas. 
LEMMA 1. Under the assumptions of the theorem, there exists a neighborhood-from-below $N$ of $P$ such that $u=M$ almost everywhere in $N$.

Lemma 2. Let $u$ be a weakly L-subcaloric function in $D$. If $u=M$ almost everywhere in a neighborhood-from-below of some point $P$ of $D$, and $M \geqq 0$, then $u=M$ almost everywhere in $C(P)$.

2. Proof of Lemma 2. We shall prove that, given a compact subset $E$ of $D$, we can construct, for each point $Q=(y, \tau)$ in $E$ a domain

$$
\Omega=\Omega_{\delta \varepsilon}:-\delta<t-\tau<0, \varepsilon|x-y|^{2}<|t-\tau|(\varepsilon>0, \delta>0)
$$

and a function $w(x, t)=w^{y, \tau}(x, t)$ having the following properties:

(a) $w>0$ in $\Omega$.

(b) $w, \partial w / \partial x_{i}, \partial^{2} w / \partial x_{i} \partial x_{j}, \partial w / \partial t$ are continuous in $\bar{\Omega}-\{(y, \tau)\}$ and vanish on the boundary $\partial \Omega-\{(y, \tau)\}$.

(c) $L^{*} w>0$ in $\Omega$.

Furthermore, $\varepsilon$ may be any number between 0 and 1 and $\delta$ may be taken to be denendent only on $L, \varepsilon$ and $E$, but not on the particular point $Q=(y, \tau)$. Finally, as $\varepsilon \rightarrow 0$, the radius of the base (or $\delta / \varepsilon$ ) can be taken to be bounded away from zero.

Once $w$ has been constructed, a simple argument of [4] can easily be extended to complete the proof of the lemma. For the sake of completeness we reproduce it here.

Let $S$ be the set of points $(x, t)$ in $C(P)$ having the property that $u=M$ almost everywhere in an open-from-below set containing $(x, t)$. By assumption $S$ is nonempty. Clearly $S$ is open from below. If we show that $S$ is also closed, then $S$ coincides with $C(P)$. To prove it, we take any sequence $Q_{m} \rightarrow R, Q_{m}$ in $S, R$ in $D$, and use the above construction with $E=\left\{R, Q_{1}, Q_{2}, \cdots\right\}$. If we show that $u=M$ almost everywhere in each domain $\Omega_{i}$ corresponding to $Q_{i}$, then it would follow that $R$ also belongs to $S$. (Note that in the construction of the $\Omega$ below, the radius of the base of $\Omega$ can be made bounded away from zero as $\varepsilon \rightarrow 0$.)

For simplicity we denote $\Omega_{i}$ by $\Omega$ and the corresponding $w_{i}$ by $w$. We now modify the definition of $w(x, t)$ in the intersection of $\Omega$ with a neighborhood-from-below $N$ of $Q_{i}$ where $u=M$ almost everywhere. The modified function is denoted by $W$, and is taken to satisfy the conditions imposed on the function $v$ in the definition of subcaloricity (in $\S 1$ ) with $E$ replaced by $\Omega$. Denote $A=N \cap \Omega, B=\Omega-A$. Using the definition of weakly $L$-subcaloric functions, we get

$$
\iint_{\Omega} u L^{*} W d x d t \geqq 0,
$$


Now,

$$
\iint_{\Omega} L^{*} W d x d t=\iint_{\Omega} W L 1 d x d t \leqq 0
$$

hence

$$
\iint_{B} L^{*} W d x d t \leqq-\iint_{A} L^{*} W d x d t
$$

On the other hand, by (2),

$$
\iint_{B} u L^{*} W d x d t \geqq \iint_{A} u L^{*} W d x d t=-M \iint_{A} L^{*} W d x d t
$$

Using (3) we obtain

$$
\iint_{B}(u-M) L^{*} W d x d t \geqq 0 \text {. }
$$

Since $L^{*} W=L^{*} w>0$ in $B, u-M$ must vanish in $B$ almost everywhere.

To complete the proof of Lemma 2 we have to construct a function $w$ which the required properties (a) - (c). For simplicity we shall do it in the special case is $\tau=0, y=0$; the general case is immediately obtained by translation.

DEFINITION OF $w$ :

$$
\begin{gathered}
w=(\delta+t)^{2}\left(-t-\varepsilon r^{2}\right)^{3} \widetilde{r}^{-k} \\
r=|x|, \widetilde{r}^{2}=r^{2}-k^{1 / 2} t
\end{gathered}
$$

where

where $k$ is a positive integer to be determined later. Clearly, $w$ satisfies (a), (b). It remains to prove that $L^{*} w>0$ in $\Omega$. We have

$$
\begin{aligned}
\frac{\partial w}{\partial x_{i}}= & -6 \varepsilon x_{i}(\delta+t)^{2}\left(-t-\varepsilon r^{2}\right)^{2} \widetilde{r}^{-k}-k x_{i}(\delta+t)^{2}\left(-t-\varepsilon r^{2}\right)^{3} \widetilde{r}^{-k-2} \\
\frac{\partial^{2} w}{\partial x_{i} \partial x_{j}}= & 24 \varepsilon^{2} x_{i} x_{j}(\delta+t)^{2}\left(-t-\varepsilon r^{2}\right) \widetilde{r}^{-k}-6 \varepsilon \delta_{i j}(\delta+t)^{2}\left(-t-\varepsilon r^{2}\right)^{2} \widetilde{r}^{-k} \\
& +12 k \varepsilon x_{i} x_{j}(\delta+t)^{2}\left(-t-\varepsilon r^{2}\right)^{2} \widetilde{r}^{-k-2}-k \delta_{i j}(\delta+t)^{2}\left(-t-\varepsilon r^{2}\right)^{3} \widetilde{r}^{-k-2} \\
& +k(k+2) x_{i} x_{j}(\delta+t)^{2}\left(-t-\varepsilon r^{2}\right)^{3} \widetilde{r}^{-k-4}, \\
\frac{\partial w}{\partial t}= & 2(\delta+t)\left(-t-\varepsilon r^{2}\right)^{3} \widetilde{r}^{-k}-3(\delta+t)^{2}\left(-t-\varepsilon r^{2}\right)^{2} \widetilde{r}^{-k} \\
& +\frac{1}{2} k^{3 / 2}(\delta+t)^{2}\left(-t-\varepsilon r^{2}\right)^{3} \widetilde{r}^{-k-2}
\end{aligned}
$$

We now form $L^{*} w$, and restrict $\delta$ to be sufficiently small and restrict $|x|$ to be sufficiently small (depending only on $L^{*}$ ), say $|x| \leqq \rho$. Then, the contribution to $L^{*} w$ made by the terms of $\Sigma b_{i} \partial w / \partial x_{i}+b w$ is small compared with the corresponding last two terms in $\partial w / \partial t$. Also, the negative contribution in $\Sigma b_{i j} \partial^{2} w / \partial x_{i} \partial x_{j}$ corresponding to the fourth 
term in $\partial^{2} w / \partial x_{i} \partial x_{j}$ (calculated above) can be neglected as compared to the third term in $\partial w / \partial t$ (provided $k$ is sufficiently large, depending on $b_{i j}$ ). Discarding (as we may) the positive contribution corresponding to the first and the last terms in $\partial^{2} w / \partial x_{i} \partial x_{j}$, we conclude that in order to prove that $L^{*} w>0$, it is sufficient to prove that

$$
k \varepsilon \frac{r^{2}}{\widetilde{r}^{2}}+k^{3 / 2}\left(-t-\varepsilon r^{2}\right) \frac{1}{\widetilde{r}^{2}} \geqq \lambda>0
$$

where $\lambda$ is a constant depending only on $L$ and $\rho(|x| \leqq \rho$ in $\Omega)$.

To prove (5) we take $k>1 / \varepsilon^{2}$, which imples that, in $\Omega$ (where $\left.\varepsilon r^{2}<|t|\right)$,

$$
k^{1 / 2}|t| \leqq \widetilde{r}^{2}=r^{2}+k^{1 / 2}|t| \leqq 2 k^{1 / 2}|t| \text {. }
$$

Hence (5) is a consequence of

$$
k^{1 / 2} \varepsilon r^{2}+k\left(-t-\varepsilon r^{2}\right) \geqq 2 \lambda|t|
$$

which is clearly true if $k^{1 / 2} \geqq 2 \lambda, k \geqq 1$.

3. Properties of fundamental solutions. Assume that the closure of a cylinder $C:|x|^{2}<\beta,-\delta<t<0$ with base $B$ is contained in $D$. By our assumptions on $L$, there exists (by Pogorzelski [7]) in $C$ a fundamental solution $\Gamma(x, t ; \xi, \tau)(t<\tau)$ of $L^{*}$ with pole $(\xi, \tau) ; L^{*} \Gamma=0$ as a function of $(x, \mathrm{t})$, and $\Gamma$ can be constructed as follows:

Let $\left(B_{i j}\right)$ be the matrix inverse to $\left(b_{i j}\right)$ and define

$$
\begin{gathered}
\sigma(x, t ; \xi, \tau)=\Sigma B_{i j}(\xi, \tau)\left(x_{i}-\xi_{i}\right)\left(x_{j}-\xi_{j}\right) \\
Z(x, t ; \xi, \tau)=(\tau-t)^{-n / 2} \exp \left\{-\frac{\sigma(x, t ; \xi, \tau)}{4(\tau-t)}\right\} \\
\Gamma(x, t ; \xi, \tau)=Z(x, t ; \xi, \tau)+\int_{t}^{\tau} \int_{B} Z(x, x ; \eta, s) \Phi(\eta, s ; \xi, \tau) d \eta d s
\end{gathered}
$$

where $\Phi$ is the solution of the integral equation

$$
\begin{aligned}
L^{*}{ }_{(x, t)} Z(x, t ; \xi, \tau) & -\rho(x, t) \Phi(x, t ; \xi, \tau) \\
& +\int_{\tau}^{t} \int_{B}\left[L_{(x, t)}^{*} Z(x, t ; \eta, s)\right] \Phi(\eta, s ; \xi, \tau) d \eta d s=0 .
\end{aligned}
$$

Here,

$$
\rho(x, t)=(4 \pi)^{n / 2} /\left(\operatorname{det}\left(B_{i j}(x, t)\right)^{1 / 2} .\right.
$$

Note that

$$
0<\text { const. } \leqq \frac{\sigma(x, t ; \xi, \tau)}{|x-\xi|^{2}} \leqq \text { const. }<\infty
$$


In the following we shall be interested in the special case $(\xi, \tau)=0$. We define

$$
\begin{aligned}
& g(x, t)=\Gamma(x, t ; 0,0) \\
& \sigma(x, t)=\sigma(x, t ; 0,0) \\
& Z(x, t)=Z(x, t ; 0,0) .
\end{aligned}
$$

By simple calculation we get

$$
g(x, t)=Z(x, t)(1+o(1))
$$

where $o(1) \rightarrow 0$ as $t \rightarrow 0$. Hence, in particular, $g(x, t)>0$ if the height $\delta$ of $C$ is sufficiently small, as we shall assume. We also mention, although this is not used later on, that for any bounded measurable function $\varphi(x, t)$ in $C$, which is continuous at $(0,0)$ we have (see [8])

$$
\lim _{t \rightarrow 0} \int_{B} g(x, t) \varphi(x, t) d x=\rho(0,0) \varphi(0,0) .
$$

We conclude this section with estimating the following expression (which will appear in the next section)

$$
I \equiv-\sum_{i, j} b_{i j}(x, t) x_{i} \frac{\partial g(x, t)}{\partial x_{j}} .
$$

Since

$$
-\frac{\partial}{\partial x_{j}} Z(x, t)=\frac{1}{2 t}\left(\sum_{k} B_{j k}(0,0) x_{k}\right) Z(x, t),
$$

and since

$$
\begin{aligned}
& \sum_{i, k} \sum_{j=1}^{n} b_{i j}(x, t) B_{j k}(0,0) x_{i} x_{k} \\
& \quad=|x|^{2}+\sum_{i, j, k}\left[b_{i j}(x, t)-b_{i j}(0,0)\right] B_{j k}(0,0) x_{i} x_{k}=|x|^{2}(1+o(1)
\end{aligned}
$$

where $o(1) \rightarrow 0$ as $|x| \rightarrow 0$, we conclude that

$$
I_{1} \equiv-\sum_{i, j} b_{i j}(x, t) x_{i} \frac{\partial Z(x, t)}{\partial x_{j}} \geqq \frac{|x|^{2}}{3|t|} Z(x, t)
$$

provided $|x|$ is sufficiently small.

To evaluate $I-I_{1}$, we use the definitions of $g$ and $\Gamma$, and proceed to estimate the $x_{j}$-derivatives of the integral which appears in the definition of $\Gamma$. Noting that

$$
\left|\frac{\partial}{\partial x_{j}} Z(x, t ; \eta, s)\right| \leqq \text { const. }(s-t)^{-1 / 2} Z(x, t ; \eta, s)
$$

and using the estimate of [7] for $\Phi$ and Dressel [1; Lemma 2] we find: that 


$$
\left|I-I_{1}\right| \leqq \lambda_{0}|x|^{\gamma} Z(x, t) \quad\left(\lambda_{0}>0,0<\gamma \leqq 1\right),
$$

where $\lambda_{0}, \gamma$ depend only on $L$. In what follows we shall only need the weaker inequality

$$
I \geqq-\lambda_{0}|x|^{\gamma} Z(x, t)
$$

4. Proof of Lemma 1. We may assume, without loss of generality, that the essential supremum $M$ is assumed at the origin. Following the procedure of Littman [4], we claim that it is enough to construct a function $G(x, t)$ in a cylinder $C:|x|^{2}<\beta,-\delta<t<0$, with base $B$, which satisfies the following conditions:

(a) $G, \partial G / \partial x_{i}, \partial^{2} G / \partial x_{i} \partial x_{\jmath}, \partial G / \partial t$ are continuous in $\bar{C}-\{(0,0)\}$ and vanish on the boundary $\partial C-\{(0,0)\}$.

(b) $L^{*} G>0$ in $C$.

(c) If $f(x, t)$ is $L$-subcaloric in a domain which contains $C$, and if $f$ is continuous from below at the origin and $f(0,0)=0$, then

$$
0 \leqq \iint_{0} f L^{*} G d x d t
$$

Once $G$ is constructed, the proof of Lemma 1 follows very easily. Indeed, $u-M$ is $L$-subcaloric, and using (c) we get

$$
\iint_{0}(u-M) L^{*} G d x d t \geqq 0 \text {. }
$$

Since, by (b), $L^{*} G>0$, we conclude that $u=M$ almost everywhere in $C$.

DEFINITION OF $G(x, t)$ :

$$
G(x, t)=(t+\delta)^{2}\left(\beta-r^{2}\right)^{3} g(x, t)
$$

where $g(x, t)$ is defined in $\S 3$. Clearly (a) is satisfied. We proceed to establish (b), (c).

Proof of $(b)$.

$$
\begin{aligned}
\frac{\partial G}{\partial x_{i}}= & -6 x_{i}(t+\delta)^{2}\left(\beta-r^{2}\right)^{2} g+(t+\delta)^{2}\left(\beta-r^{2}\right)^{3} \frac{\partial g}{\partial x_{i}} \\
\frac{\partial^{2} G}{\partial x_{i} \partial x_{j}}= & -6 \delta_{i j}(t+\delta)^{2}\left(\beta-r^{2}\right)^{2} g+24 x_{i} x_{j}(t+\delta)^{2}\left(\beta-r^{2}\right) g \\
& -6 x_{i}(t+\delta)^{2}\left(\beta-r^{2}\right)^{2} \frac{\partial g}{\partial x_{j}}-6 x_{j}(t+\delta)^{2}\left(\beta-r^{2}\right)^{2} \frac{\partial g}{\partial x_{i}} \\
& +(t+\delta)^{2}\left(\beta-r^{2}\right)^{3} \frac{\partial^{2} g}{\partial x_{i} \partial x_{j}},
\end{aligned}
$$




$$
\frac{\partial G}{\partial t}=2(t+\delta)\left(\beta-r^{2}\right)^{3} g+(t+\delta)^{2}\left(\beta-r^{2}\right)^{3} \frac{\partial g}{\partial t} .
$$

Recalling that $L^{*} g=0$ we obtain

$$
\begin{aligned}
L^{*} G= & -6(t+\delta)^{2}\left(\beta-r^{2}\right)^{2} g \Sigma x_{i} b_{i}-6\left(\Sigma b_{i i}\right)(t+\delta)^{2}\left(\beta-r^{2}\right)^{2} g \\
& +24\left(\Sigma b_{i j} x_{i} x_{j}\right)(t+\delta)^{2}\left(\beta-r^{2}\right) g-12(t+\delta)^{2}\left(\beta-r^{2}\right)^{2} \Sigma b_{i j} x_{i} \frac{\partial g}{\partial x_{j}} \\
& +2(t+\delta)\left(\beta-r^{2}\right)^{3} g .
\end{aligned}
$$

Now the first term in $L^{*} G$ is small compared with the second one, if $|x|$ (or $\beta$ ) is small. Using (8), (10), (6) to estimate the fourth term, we conclude that, if it is negative, then its absolute value is smaller than that of the second term. Hence, if we prove that

$$
(t+\delta) r^{2}+\left(\beta-r^{2}\right)^{2}>\mu(t+\delta)\left(\beta-r^{2}\right)
$$

for sufficiently large $\mu$ depending only on $L$ (provided $\beta$ is smaller than an appropriate constant), then $L^{*} G>0$.

To prove (13) we note that if $\mu\left(\beta-r^{2}\right)<r^{2}$ then (13) clearly holds. Hence it remains to consider the case where

$$
\mu\left(\beta-r^{2}\right) \geqq r^{2} .
$$

However, in this case

$$
\left(\beta-r^{2}\right)^{2} \geqq \frac{\beta^{2}}{(1+\mu)^{2}}>\mu(t+\delta) \beta
$$

for sufficiently small $\delta$ (i.e., if $(\mu+1)^{2} \mu \delta<\beta$ ), from which (13) follows.

Proof of (c). We modify $G$ as follows: Let

$$
\sigma_{\varepsilon}(x, t)=\left\{\begin{array}{l}
\sigma(x, t) \text { if }-\delta<t<-\varepsilon \\
\sigma(x, t)+(t+\varepsilon) \text { if }-\varepsilon \leqq t \leqq 0 .
\end{array}\right.
$$

Clearly $\sigma_{\varepsilon}(x, t)$ has second continuous $x$-derivatives and a first continuous $t$-derivative in $\bar{C}$. We next define

$$
\begin{aligned}
& Z_{\varepsilon}(x, t)=\frac{1}{(-t)^{n / 2}} \exp \left\{\frac{\sigma_{\varepsilon}(x, t)}{4 t}\right\} . \\
& g_{\varepsilon}(x, t)=Z_{\varepsilon}(x, t)+\int_{t}^{0} \int_{B} Z(x, t ; \eta, s) \Phi(\eta, s ; 0,0) d \eta d s, \\
& G_{\varepsilon}(x, t)=(t+\delta)^{2}\left(\beta-r^{2}\right)^{3} g_{\varepsilon}(x, t) .
\end{aligned}
$$

$G_{\varepsilon}$ is differentiable also at the origin where it vanishes. We now proceed to prove (c).

By the definition of $L$-subcaloricity (see (1)) we have, 


$$
\iint_{\sigma} f(x, t) L^{*} G_{\varepsilon}(x, t) d x d t \geqq 0 .
$$

If we prove that

$$
\begin{aligned}
& \left.\lim _{\varepsilon \rightarrow 0} \iint_{O_{\varepsilon}} f x, t\right) L^{*} G(x, t) d x d t=0, \\
& \lim _{\varepsilon \rightarrow 0} \iint_{\sigma_{\varepsilon}} f(x, t) L^{*} G_{\varepsilon}(x, t) d x d t=0,
\end{aligned}
$$

where $C_{\varepsilon}=C \cap\{-\varepsilon<t<0\}$, then (c) follows from (14).

In what follows we denote any positive constant (independent of $\varepsilon$ ) by the same symbol $A$. To prove (15) we write

$$
\iint_{\sigma_{\varepsilon}} f L^{*} G d x d t=\int_{-\varepsilon}^{0} \int_{|x|<\eta} f L^{*} G d x d t+\int_{-\varepsilon}^{0} \int_{\eta<|x|<\beta} f L^{*} G d x d t,
$$

where $\eta$ is any positive number smaller than $\beta$. Since $f$ is continuous from below at $(0,0)$ and $f(0,0)=0$, the first integral on the right side of (17) tends to zero as $\eta \rightarrow 0$, independently of $\varepsilon$.

Here we have made use of (see [7])

$$
\left|L^{*} G(x, t)\right| \frac{A}{|t|^{(n+\nu+1) / 2}} \exp \left\{\frac{A r^{2}}{t}\right\} \text { for some } 0 \leqq \nu<1 .
$$

The second integral on the right side of (17), for any fixed $\eta$, also tends to zero as follows by using (18).

Proof of (16). Proceeding similarly to the proof of (15), we find that all that remains to be proved is that

$$
\iint_{C_{\varepsilon}}\left|L^{*} G_{\varepsilon}\right| d x d t \leqq A<\infty
$$

for all $\varepsilon>0(A$ is independent of $\varepsilon)$. Now,

$$
\begin{gathered}
-L^{*} g_{\varepsilon}=L^{*}\left(g-g_{\varepsilon}\right)=L^{*}\left(Z-Z_{\varepsilon}\right)=L^{*}\left[Z(x, t)\left(1-\exp \left\{\frac{(t+\varepsilon)^{2}}{4 t}\right\}\right)\right] \\
=L^{*} Z\left(1-\exp \left\{\frac{(t+\varepsilon)^{2}}{4 t}\right\}\right)-Z\left[\exp \left\{\frac{(t+\varepsilon)^{2}}{4 t}\right\}\right]\left[\frac{t+\varepsilon}{2 t}-\frac{(t+\varepsilon)^{2}}{4 t^{2}}\right] .
\end{gathered}
$$

Since

$$
\left|L^{*} Z\right| \leqq \frac{A}{|t|^{(n+1+\gamma) / 2}} \exp \left\{\frac{A r^{2}}{t}\right\}
$$

for some $0 \leqq \nu<1$, we find, denoting $\left|\frac{t+\varepsilon}{2 t}-\frac{(t+\varepsilon)^{2}}{4 t^{2}}\right|$ shortly by $[\cdots]$,

$$
\begin{aligned}
\left|L^{*} G_{\varepsilon}\right| \leqq & \frac{A}{|t|^{(n+1+\nu) / 2}} \exp \left\{\frac{A r^{2}}{t}\right\} \\
& +\frac{A}{|t|^{n / 2}}[\cdots] \exp \left\{\frac{A r^{2}}{t}\right\} \exp \left\{\frac{(t+\varepsilon)^{2}}{4 t}\right\} \equiv K_{1}+K_{2} .
\end{aligned}
$$


The integral of $K_{1}$ is easily seen to be bounded. Hence it remains to evaluate

$$
J \equiv \iint_{O_{\varepsilon}} K_{2} d x d t
$$

We split $J$ in the following way:

$$
J=\int_{-\varepsilon}^{-\varepsilon / 2} \int_{B} K_{2} d x d t+\int_{-\varepsilon / 2}^{0} \int_{B} K_{2} d x d t \equiv J_{1}+J_{2} .
$$

As for $J_{1},[\cdots] \leqq 1$ and hence $J_{1} \leqq A$. As for $J_{2},[\cdots] \leqq A \varepsilon^{2} / t^{2}$ and hence

$$
J_{2} \leqq A \varepsilon^{2} \int_{-\varepsilon / 2}^{0}\left(\int_{B} \frac{1}{|t|^{n / 2}} \exp \left\{\frac{A r^{2}}{4 t}\right\} d r\right) \frac{1}{t^{2}} \exp \left\{\frac{A \varepsilon^{2}}{t}\right\} d t
$$

The inner integral is bounded. Substituting $z=\varepsilon^{2} /|t|$ we get

$$
J_{2} \leqq A \int_{2 \varepsilon}^{\infty} e^{-A z} d z
$$

We have thus proved that $J=J_{1}+J_{2} \leqq A$, which completes the proof of (19). Hence, the proof of (16) is completed.

REMARK. The maximum principle for subelliptic functions [4] follows from the maximum principle for subparabolic functions proved in this paper. Indeed, as is easily seen, a weak subelliptic function is necessarily a weak subparabolic function.

\section{REFERENCES}

1. F.G. Dressel, The fundamental solution of the parabolic equation II, Duke Math. J., 13 (1946), 61-70.

2. A. Friedman, Parabolic equations of the second order, Trans. Amer. Math. Soc., 93 (1959), 509-530.

3. E. Hopf, Elementare Bemerkungen über die Lösungen partieller Differentialgleichungen zweiter Ordnung von elliptischen Typus, Sitzungsberichte, Berlin Akad. Wiss., 19 (1927), 147-152.

4. W. Littman, A strong maximum principle for weakly L-subharmonic functions, J. Math. and Mech., 8 (1959), 761-770.

5. L. Nirenberg, A strong maximum principle for parabolic equations, Comm. Pure App. Math., 6 (1953), 167-177.

6. — Existence Theorems in Partial Differential Equations, New York University Notes.

7. W. Pogorzelski, Étude de la solution fondamentale de l'équation parabolique, Ricerche di Mat., 5 (1956), 25-57.

8. — Propriétés des intégrales de l'équation parabolique normale, Ann. Polon, Mat., 4 (1957), 61-91.

University of Minnesota, Institute of Technology, Minneapolis, Minnesota 


\section{PACIFIC JOURNAL OF MATHEMATICS}

\section{EDITORS}

Ralph S. Phillips

Stanford University

Stanford, California

F. H. BrownelL

University of Washington

Seattle 5 , Washington
A. L. Whiteman

University of Southern California Los Angeles 7. California

L. J. PAIGE

University of California

Los Angeles 24, California

ASSOCIATE EDITORS
E. F. BECKENBACH
D. DERRY
H. L. ROYDEN
E. G. STRAUS
T. M. CHERRY
M. OHTSUKA
E. SPANIER
F. WOLF

\section{SUPPORTING INSTITUTIONS}

UNIVERSITY OF BRITISH COLUMBIA

CALIFORNIA INSTITUTE OF TECHNOLOGY

UNIVERSITY OF CALIFORNIA

MONTANA STATE UNIVERSITY

UNIVERSITY OF NEVADA

NEW MEXICO STATE UNIVERSITY

OREGON STATE COLLEGE

UNIVERSITY OF OREGON

OSAKA UNIVERSITY

UNIVERSITY OF SOUTHERN CALIFORNIA
STANFORD UNIVERSITY

UNIVERSITY OF TOKYO

UNIVERSITY OF UTAH

WASHINGTON STATE COLLEGE,

UNIVERSITY OF WASHINGTON

AMERICAN MATHEMATICAL SOCIETY

CALIFORNIA RESEARCH CORPORATION

HUGHES AIRCRAFT COMPANY

SPACE TECHNOLOGY LABORATORIES

NAVAL ORDNANCE TEST STATION

Printed in Japan by International Academic Printing Co., Ltd., Tokyo, Japan

Reprinted 1966 in the United States of America 


\section{Pacific Journal of Mathematics}

\section{Vol. 11, No. $1 \quad$ November, 1961}

A. A. Albert, Generalized twisted fields ............................ 1

Richard Arens, Operational calculus of linear relations ................... 9

John Herbert Barrett, Disconjugacy of a self-adjoint differential equation of the fourth order ....................................... 25

Paul Richard Beesack, Hardy's inequality and its extensions ............... 39

Julius Rubin Blum and David Lee Hanson, On invariant probability measures.

II .............................................

Robert Allen Bonic, Symmetry in group algebras of discrete groups.......... 73

R. Creighton Buck, Multiplication operators ...................... 95

Jack Gary Ceder, Some generalizations of metric spaces ................. 105

Meyer Dwass, Random crossings of cumulative distribution functions ......... 127

Albert Edrei, Wolfgang H. J. Fuchs and Simon Hellerstein, Radial distribution and

deficiencies of the values of a meromorphic function ............... 135

William Cassidy Fox, Harmonic functions with arbitrary local singularities ..... 153

Theodore Thomas Frankel, Manifolds with positive curvature ............... 165

Avner Friedman, A strong maximum principle for weakly subparabolic

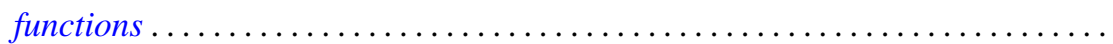

Watson Bryan Fulks and J. O. Sather, Asymptotics. II. Laplace's method for

multiple integrals ......................................

Adriano Mario Garsia and Eugene Richard Rodemich, An embedding of Riemann

surfaces of genus one ..................................... 193

Irving Leonard Glicksberg, Weak compactness and separate continuity......... 205

Branko Grünbaum, On a conjecture of H. Hadwiger .................. 215

Frank J. Hahn, On the action of a locally compact group on $E_{n} \ldots \ldots \ldots \ldots \ldots . . \ldots 221$

Magnus R. Hestenes, Relative hermitian matrices ..................... 225

G. K. Kalisch, On similarity invariants of certain operators in $L_{p} \ldots \ldots \ldots \ldots .247$

Yitzhak Katznelson and Walter Rudin, The Stone-Weierstrass property in Banach

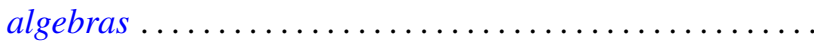

Samir A. Khabbaz, The subgroups of a divisible group $G$ which can be represented as intersections of divisible subgroups of $G \ldots \ldots \ldots \ldots \ldots \ldots \ldots \ldots \ldots . \ldots \ldots 7$

Marvin Isadore Knopp, Construction of a class of modular functions and forms .......................................... 275

Charles Alan McCarthy, Commuting Boolean algebras of projections .......... 295

T. M. MacRobert, Transformations of series of E-functions ................ 309

Heinz Renggli, An inequality for logarithmic capacities ................. 313

M. S. Robertson, Applications of the subordination principle to univalent functions .......................................... 315

David Sachs, Partition and modulated lattices ..................... 325

Frank S. Scalora, Abstract martingale convergence theorems ............... 347

Elbert A. Walker, Torsion endomorphic images of mixed Abelian groups ........ 375

Morgan Ward, The prime divisors of Fibonacci numbers................. 379

Charles R. B. Wright, On the nilpotency class of a group of exponent four....... 387 